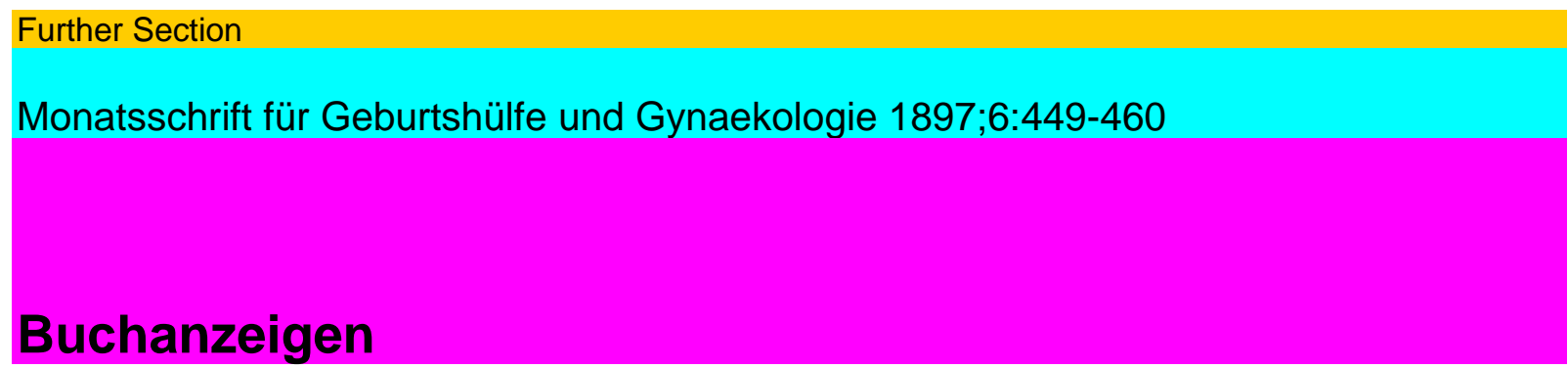

\title{
H. Hegar-Kaltenbach
}

Operative Gynäkologie mit Einschluss der gynäkologischen Untersuchungslehre.. 4. umgearbeitete und ver-mehrte Auflage. Herausgegeben von A. He gar unter Mitwirkung von $\Lambda$ V. $\Lambda$ Viedow, E. Sonntag, G. Bulius. Stuttgart, Verlag von F. Enke. 1897.

$\Lambda$ Venn von einem allseitig als klassisch anerkannten Werke eine neue Auflage erscheint. $\Lambda$ venn $\mathrm{zu}$ diesem, von einem Hegar herausgegebenen Buche ein $\Lambda V$. A. FreundJ) sich schon geäussert hat, dann kann eine nach-folgende Besprechung nur die Aufgabe haben, aucli den Aerzten, welchen ein eingehendes Studium des Buches aus irgend welchen Gründen nicht möglich ist, den reichen Inhalt einigermassen zugänglich zu machen, be-sonders aber diejenigen Punkte hervorzuheben, die sich gegen früher geändejrt oder die besonders zur Diskussion gestanden haben.

Körperpositionen. Die für die Gynäkologie so wichtig gewordene Beckenhochlagerung findet eingehende Besprechung, ihre grossen Vorzüge $\Lambda$ verden gerühmt, ihre Nachteile nicht verschwiegen, doch gewiss mit Recht als grösstenteils zu umgehen bezeichnet. Viel ausführlicher als früher ist die Narkose behandelt; die grundsätzliche Untersuchung auch des Harns vor derselben wird nachdrücklichst verlangt; der lokalen Anästhesie, der Anwendung des Aethers, der Chloroformtropfenmethode wird gedacht, ohne dass denselben Bedeutung beigelegt wird. Die Freiburger Klinik ist der alt erprobten An $\Lambda$ vendungsweise des Chloroforms treu geblieben.

Bei der Besprechung der kombinirten Untersuchung werden (bes. gegenübor A. Martin) mit erneutem Nachdruck die $\Lambda$ Torteile des rectoabdominalen $\Lambda \Gamma$ orgehens hervorgehoben.

Lehrevonden gynäkologischen Operationen. Die intraute-rinen Inj ectionen werden gegenüber Fehling und Saenger als w $3 / 8$ ksamste Methode intrauteriner Therapie aufrecht erhalten, allerdings unter ganz be-sonderer Betonung der nötigen $\Lambda \tau$ orsichtsmassregeln; für die fíüssigen Substanzen wird die Saenger'sche Aetzskala angeführt, die Anwendung der Medikamente in fester Form nur des historischen Interesses halber er-wähnt. Die Pess artherapie ist ausführlich besprochen; bei Prolaps halt sie Hegar bekanntlich nur dann für indizirt, wenn lokale oder all-

i) Oentralblatt f. Gyn. 1897, No. 19.

450

Buchanzeigen.

gemeine Krankheitszustände die Operation durchaus verhindern; bezüglich der Lageveränderungen des Uterus sei erwähnt, dass intrauterine Pessarien seit mehr als einem Jahrzehnt nicht mehr angewendet $\Lambda$ vurden.

Das neu eingefügte Kapitel über elektrische Behandlung ist sehr kurz ausgefallen, $\Lambda$ vas die meisten deutschen Fachgenossen wohl nicht als Mangel empfinden werden; He gar hat dieselbe nicht angewendet; ausführ-licher behandelt ist die Massage und Thure Brandt's Verfahren; erstere wird (wie früher) empfohlen für Subinvolutio uteri, weiterhin auch für starre Exsudate im Bindegewebe, Beckenbauchfell. in den Lig. sacrouterinis; be-züglich der Ovarien und Tuben $\Lambda$ vird eher von ihrer Anwendung abgeraten. Für das eigentliche Brandt'sche Verfahren vermisst 
Hegar die physio-logische Grundlage, auf welche hin man bestimmte "Wirkungen auch nur halbwegs sicher erwarten könnte.

In dem Kapitel „, $\Lambda$ Vundnaht” zeigt sich, dass Hegar seinem Grund-satz treu geblieben ist, bei plastischen Operationen im Allgemeinen nur soviel anzufrischen, dass die Vereinigung durch einfache Suturen ohne zu grosse Mühc gelingt; er betrachtet daher die versenkte Catgutnaht nach wie vor nur als ein, im gegebenen Fall freilich vortreffliches Aushilfsmittel; die der fortlaufenden Catgutnaht später zugeschriebenen Nachteile werden jeden-falls nicht allerseits anerkannt, so wird z. B. die Cutis bei Laparotomien wohl nicht nur an unserer Klinik ebenfalls mit fortlaufendem Catgut vernäht - mit bestem Erfolge. Die Schwierigkeit oder wenigstens Umständlichkeit der Sterilisation muss freilich zugegeben werden. Den neuen Vorschlägen Walcher's, die Faltung der Wundränder durch kreisförmige Umstechung derselben zu umgehen, wird keine Bedeutung beigelegt, bezüglich der Bauch-naht Walcher's Verfahren aus praktischen und theoretischen Gründen direkt verworfen; sehr wesentlich scheint uns die dabei angebrachte Warming vor zu festem Schnüren der Nähte; es wird dabei auf experimentelle, noch nicht veröffentlichte Untersuchungen Tilgler's über die $\Lambda$ Vundnaht Bezug genommen. In der leichten Vermeidung zu starker Schnûrung sieht Hegar auch einen Hauptvorteil der von ihm so bevorzugten Drahtnaht.

Im Kapitel Antisepsis und Asepsis sind die jetzt allgemein üb-lichen Maassnahmen sehr anschaulich zusammengefasst, ich hebe nur hervor $\tau$ dass, $\Lambda$ venigstens nach Berührung mit sehr infektiösem Material, Abstinenz gefordert wird. Der Gebrauch der Schwämme ist fast vollständig auf-gegeben.

Als sehr vorteilhafte Aenderung gegenüber der älteren A ullage er-weist sich die klare und knappe Zusammenfassung aller auf die vom Abdomen her ausgeführten Operationen bezüglichen, allgemeinen Gesichtspunkte unter dem Titel Laparotomie.

Die Bauchnaht wird so ausgeführt, dass zuerst das Peritoneum allein oder mit der Fascie durch Catgutknopfnähte, dann die übrigen Abschnitte der Bauchdecken durch tiefe und oberflächliche Seidensuturen geschlossen werden; in die Zwischenrãume zwischen die Nähte kommen kleine sterilisirte Gazestreifen zur Drainage, welche am 2.-3. Tage entfernt werden. Für die Bauchhöhle ist die prophylaktische Drainage für besondere Verhältnisse bei-behalten (Verletzungen von Darm und Ureter, Austritt infektiösen Materials, unvollständige Blutstillung); die Herausleitung der Drains durch die Scheide wird bevorzugt. Bezüglich der Nachb ehandlung wird besonders ein-dringlich vor dem „zu viel thun” gewarnt. Häufig wird eine schiefe-Lagerung der Operirten mit dem Kopf nach unten angewendet und davon auch ein Ausbleiben etwa zu furchtender Verklebungen der Därme mit Wundflächen im Becken erwartet. Zur Vermeidung von Herzkollaps und namentlich Thrombosen. wird eine besondere Kräftigungskur vor der Operation angeraten; nach der letzteren werden $\Lambda$ Veinklystiere, möglichst frühzeitige passive Bewegungen der Beine empfohlen, vor einer zu starken Compression des Abdomen gewarnt.

Bei Darmocclusion gelang es Hegar (neben den zwei schon früher berichteten) in zwei neuen Fallen, durch erneute Laparotomie Genesung zu erzielen; der Darm war an das Schnürstück nach Ovariotomie angeíötet gewesen.

Buchanzeigen.

451

Die $0 \backslash \cdot$ ari o torn ie stellt ein im Ganzen so abgekliirtes Gebiet dar, dass icli mich mit der Hervorhebung weniger Punkte begnügen kaim. Bei den noch im kleinen Becken liegenden Tumoren bedarf es ausser der An-wesenheit der Geschwulst noch einer besonderen Indikation, um sofort zur Operation zu schreiten. Bei bestehender Schwangerschaft ist frühzeitig zu 
operiren, am besten im 2.-4. Monat, wobei sich in 85-90 pCt. ein normaler weiterer Schwangerschaftsverlauf ergab. Die Resektion des eventuell partiell erkrankten zweiten Ovarium wird ziemlich absprechend beurteilt; dagegen soil jedes „,bedeutend”, wenn auch nicht cystisch veränderte II. Ovarium ganz entfernt werden. Die einzelnen die Prognose be-stimmenden Momente $\Lambda$ verden auseinandergesetzt, dagegen mit Recht bemerkt, dass die Statistikeu mit ihren Gesammtzahlen ohne Berûcksichtigung der Einzelheiten in unserer Zeit wenig Wert mehr haben; doch wird die interessante Tabelle Péan's angeführt, die über 7 Serien von Ovariotomien von 1864-1887 berichtet mit einem Herabsinken der Mortalität von 24,4 auf 2,1 (bezw. auf 0,0) pCt. Der Ovariotomia vaginalis wird für die ge-wöhnlichen Fälle keine grosse Zukunft prophezeit. Die Punction wurde seit $1 \delta$ Jahren nicht mehr ausgeführt; sie ist nur bei absoluter Contraindication der Operation zulässig, sonst auch nicht einmal zu diagnostischen Zwecken. Nach Cast ration (doppelseitiger) trat von 167 Fallen die Menopause nur in 55,7 pCt. sofort, in 25,1 pCt. nach ein- oder mehrmaligem Blut-abgang ein, in 19,2 pCt. war sie ein Jahr nach der Operation noch nicht vorbanden. Die „Ausfallserscheinungen” werden nicht sehr hoch angeschlagen, da das Allgemeinbeñnden der Operirten durch dieselben niemals in nennenswerter $\Lambda$ Veise gestört war; es wird daher gegenüber Werth die möglichst vollständige Entfernung der Ovarien verlangt; auch die Schaffung einer Praedisposition zu Psychosen durch die Castration (Glaevecke) wird nicht anerkannt.

Die allgemeine Begründung des bekannten Hegar'schen Standpunktes in der Castrationsfrage hat eine wesentliche Aenderung nicht erfahren. Von der Vorbedingung der vorherigen Abtastung der Eierstöcke, der Feststellung ihrer Beschaffenheit und Insertion darf nur bei Fibroin en und Osteomalacie Abstand genommen werden; bei ersterer ist die Castration noch immer der Operation der Wahl und der Totalexstirpation und Amputatio supravaginalis vorzuziehen, abgesehen von bestimmten Ausnahmen (kolossale, fibrocystische, adenomyomatöse, gestielte Tumoren); die von Bulius festgestellte abnorme Beschaffenheit der Ovarien bei Myome $\alpha$ wird besonders betont. Bezüglich der Osteomalacie wird WinckeГs Zurückhaltung nicht geteilt, vielmehr geraten, nicht gar zu viel Zeit mit dem Ausprobiren anderer Mittel zu ver-lieren. Als anatomischer Befund der Ovarien bei derselben wird eine viel-fach zu ßlutextravasaten führende Hyperaemie mit Vermehrung und Er- $\Lambda$ veiterung der Gefässe, sowie hyaline Entartung der Arterien beschrieben.

Aus der genauen Besprechung der übrigen zur Castration Anlass gebenden anatomischen Befunde ergiebt sich, dass es auf den einschlägigen, früher strittigen Gebieten (Ziffer 1, 5, 6) leider auch in den letzten 11 Jahren nicht gelungen ist für den Einzelfall strengere, objektive, vor der Operation feststellbare Anhaltspunkte aufzufinden dafür, ob man bestïmmte Befunde als pathologisch erklären soil und in welchem Umfang dieselben dann verant-wortlich gemacht werden dürfen für vorhandene Symptome, namentlich all-gemeiner Natur.

Die Mortalität der Operation ist von 12,1 auf 4,3 pCt. (in 139 von 1885-1894 operirten Fallen) gesunken. Die Dauererfolge waren sehr günstig 1/8ei Myom und Osteomalacie, sehr ungleichmässig bei den Menstruations-anomalien, recht zweifelhaft bei den entzündlichen Zuständen.

Operationen an den Tuben. Bei Hydrosalpinx wird die Operation von den Beschwerden abhängig gemacht, bei Pyosalpinx soil nach gestellter Diagnose stets operirt werden, am besten, wenn die Infektiosität des Inhalts erloschen ist, also etwa ein Jahr nach der Entstehung, doch lasse sich die Operation nicht immer so lange hinausschieben. Auch die Rücksichtnahme auf die Lebensstellung der Patientin wird mit Recht hervorgehoben. Die Drainage nach cler Operation wurde in den letzten Jahren nicht mehr aus- 
452

Buchanzeigen.

geführt, doch wird sie nicht vollständig verworfen, sondern für besondere Verhältnisse (s. o.) empfohleu. Die Prognose bez. Beseitigung der Be-sehwerden ist mit Vorsicht zu stellen, nur bei Tuberculose wurden sehr gute Erfolge erzielt.

Die Exstirpation tubarer Fruchtsäcke wird, bei der Häufigkeit der späteren Heilung dieses Zustandes, in den ersten Monaten nicht ohne weiteres, sondern nur dann für indizirt gehalten, wenn die Operation vor-aussichtlich leicbt ist, wenn gefährliche Zufälle eintreten oder wenn die Kranken sich niclit ruhig verhalten und unter ärztlicher Ueberwachung bleiben können. Dagegen soil in der zweiten Hälfte der Schwangerschaft baldmögliclist operirt werden.

Eine weitgehende Umarbeitung hat das Capitel über Totalexstir-pation des Uterus erfahren. Bei abdominaler Operation, die vorzüglich für Myome in Frage kommt (obwobl gegenüber der supravaginalen Amputation die längere Dauer für die meist sehr anämischen Kranken ungünstig ist) soil im wesentlichen der Gang der modifizirten Freund'schen Methode befolgt werden, bei der ,,der wichtigsten Vorbedingung für einen güøstigen Aus-gang”, dem vollkommenen Abschluss der Bauchhöhle Rechnung zu tragen ist; also Umschneidung des Collum und Versorgung der Art. uterina von der Scheide aus, dann erst Laparotomie, Unterbindung der Lig. lata, Bilduug der peritonealen Lappen, Loslösung der Blase, symperitoneale Naht nach Einstülpung der Stümpfe und Ligaturen in die Scheide. Die Modificationen von A. Martin, Doyen, Richelot, Péan werden kurz und klar geschildert.

Die vaginale Operation wird in 4 Akten beschrieben. I. Umschneidung des Scheidengewölbes, zuerst hinten mit sofortiger Eröffnung des Douglas. II. Loslösen der Blase, Eröffnung der Fossa vesico-uterina. III. Loslösen der seitíichen Verbindungen, Unterbindung der Lig. lata, event. mit Umstürzen des Uterus nach D $\pi$ rchtrennung der unteren Hälfte der Ligamente. IV. Schluss der Scheidenbauchwunde. Die Verfahren anderer Operateure werden, zum Teil in knapp referirender Form, beschrieben, so namentlich die Klemm- und Verkleinerungsmethode nach Péan und D oyen-Landau, das Verfahren von Fritsch bei Prolaps, von Leopold, Richelot und Anderen bei Myom, von Péan, Segond, Landau bei entzündlichen Veränderungen der Acínexe, schliesslich die sacralen, parasacralen und perinealen Methoden.

Für das Carcinoma uteri ist die vaginale Operation noch immer als bevorzugtes Verfahren anzusehen, während die sacrale Methode bei höherer Mortalität (182) die auf sie gesetzten Hoffnungen nicht erfüllt hat. Für alle Methoden gilt die Einschränkung der Operation auf Fälle, in denen das Beckenbindegewebe noch nicht in ausgedehntem Maasse ergriffen ist. Die Vorzüge der Klemm-Methode bei fixirtem Uterus werden anerkannt, die kurze Operationsdauer, die Möglichkeit, die Klemmen weiter lateralwärts anzulegen, die sichere Blutstillung hervorgehoben; „einem abfälligen Urtheil” vermag sich daher die Freiburger Klinik nicht anzuschliessen. Bei Myom bis zu Kindskopfgrösse wird der Castration vor der etwa anwendbaren vaginalen Totalexstirpation der Vorzug gegeben, ausser bei festen Verwachsungen der Ovarien oder submucösem und intraligamentärem Sitz der Geschwulst. Die ventrale Totalexstirpation ist bei entsprechender Grösse des Tumors vorderhand nur zu wählen bei vorwiegend intraligamentärer Ent- $\Lambda$ vicklung, $\Lambda$ veil nach Entfernung der Cervix die grosse Wundhöhle im Para-metrium besser nach der Scheide drainirt werden kann. Bei entzündlichen Adne xerkrankungen mit Eiterung wird der Totalexstirpation des Uterus nicht geradc das Wort geredet, doch bilde sie in gewissen Fallen das ultimum refugium; noch vorsichtiger ist die Indíkation für ent-zündliche Prozesse ohne Eiterung formulirt; $\Lambda$ vegen Prolaps soil die Operation nur bei bestehenden Complikationen ausgeführt $\Lambda$ verden. 
Die für Myomotomie aufgeführten Indikationen sind die allgemein anerkannten; bezüglich der Complikation mit Schwangerschaft teilt Hegar Hofmeier's Standpunkt: Die Operation soil im Interesse des Kindes so lange hinausgeschoben $\Lambda$ verden, bis wii $\cdot k l i c h$ Gefahr für die Mutter eintritt; die künstlíche Unterbrechung der Scl $\pi$ vangerschaft kommt nicht in Frage. BeBuchanzeigen.

458

züglich der Technik haben sich nur bei der intraperitonealen Methode der Stielversorgung Aenderungen ergeben; nacli genauer Schilderung der ur-sprünglichen Schr o e der'schen Methode wird als wichtige Verbesserung derselben die Uebernähung des Stnmpfes mit grossen Peritoneallappen nach Hofmeier und Chrobak, besonders wegen des sicheren Abschlusses der Amputationsfläche gegen die Bauchhöhle hervorgehoben, wegen der exakten Blutstillung die Zweif el'sche Methode der fortlaufenden Kettennaht gerühmt.

Bei der Kritik der Methoden wird immer die Statistik als wertlos bezeichnet; übrigens zeigt eine nach verschiedenen Autoren zusammen-gestellte Tabelle eine Abnahme der Mortalität bei extraperitonealer Be-handlung von 50 auf 8,3 pCt., bei intraperitonealer von 73,8 auf 9,54 pCt., beides in den Jahren 1875-1895. Trotzdem darnach die Prognose ziemlich gleich erscheint, wird doch die extraperitoneale Methode Hegar's noch vorgezogen wegen des absolut sicheren Schutzes vor Blutung und der minimalen Gefahr nachträglicher Infektion; den neueren Methoden der intraperitonealen Versorgung $\Lambda$ vird zugestanden, dass die eine Hauptgefahr, die ungenügende Blutstillung, fast vollständig beseitigt sei, die andere je-doch, die Möglichkeit unmittelbarer oder nachträglicher Infektion wird noch sehr hoch - unserer Meinung nach zu hoch - angeschlagen. Dem Satze freilich, dass trotz aller bakteriologischen Untersuchungen der Keimfreiheit des Uterus und oberen Cervixteils nicht immer zu trauen ist, wird man zu-stimmen müssen. Als sehr bedenklich wird das Versenken der elastischen Ligatur bezeichnet, die A. Martin'sche Enucleation als zu gefährlich (unmittel-bare und nachträgliche Blutung und Eiterung) verworfen. Die vaginale Myomotomie nach Dührssen-Martin oder Doyen-Veít wird für kleinere Geschwulste bis zu Faustgrösse, sofern sie solitär sind, anerkannt, sonst die vaginale Totalexstirpation bevorzugt.

Die Amputatio colli wird auch bei Portiocancroid ganz im Beginne als berechtigt erklärt, trotz der mehrfachen Einwendungen; doch wird die grosse Seltenheit hierhergehöriger Fälle betont. Nicht mehr anerkannt als Anzeige der vaginalen Amputation wird die Metritis chronica; die angeb-liche Rückbildung des Corpus uteri nach dieser Operation sei weder klinisch noch anatomisch festgestellt.

Bei der Enucleation von My omen von der Scheide aus wird die Verbesserung der Prognose (3,4 pCt. Mortalität gegen 16 in älteren Zu-sammenstellungen) im wesentlichen der Beschränkung auf passende Fälle zu-geschrieben, das sind neben den leicht zugängigen Cervixmyomen die solitären, locker eingebetteten, die oberen Teile der Cervix zum Verstreichen bringenden, nicht allzu grossen Tumoren des Corpus; auch jetzt noch wird also die Indikation der Operation viel enger gestellt als von den französischen Autoren und vor den Gefahren - Blutung und Sepsis eindringlich gewarnt.

Die neuen Retroflexionsoperationen werden eingeteilt in I. solche, welche durch Yerkürzung der natürlichen Befestigungsmittel des Uterus Heilung bezwecken, II. solche, welche den Uterus durch neue künstliche Befestigungen nach vorne zu halten suchen. Die Alexander'sche Operation erfordert, um Misserfolge zu vermeiden, stets eine vollständige Spaltung des Leistenkanals nach Kocher, bei dem ursprünglichen Alexander'schen Verfahren wird häufig das an der allgemeinen Atrophie der Befestigungsmittel teilnehmende Lig. rot. nicht gefunden; das 
Kocher'sche Verfahren aber wurde als ein gar zu grosser Eingriff und wegen der Gefahr der Brüche nicht ausgeführt. Die Ventrofixation ist das bessere, sicherere, bei fixirter Eetroflexio das einzig zulässige Verfahren, naihentlich auch $\Lambda$ vegen der Uebersichtlichkeit und sicheren Blutstillung gegenüber der Vaginofixation, die bei zeugungsfähigen Frauen wegen der sch $\lambda$ veren Ge-burtsstörungen überhaupt contraindizirt ist. Die Czerny'sche Methode der Ventrofixation (Durchführung der Nähte durch den Fundus selbst, durch die Bauchdecken nur bis zur Fascie) $\Lambda$ vird vor der Olshausen's und Leopold's bevorzugt. Die Vaginofixation wird in Freiburg so ausgeführt, dass nach Eröffnuug des Peritoneum der Fundus durch eine einzige Péan-sche Kugelzange gefasst und vorgezogen und dann durch Seidenfäden, welche 454

Buchanzeigen.

die Scheidenwände niclit durchsetzen, ini vorderen Wundwinkel befestigt wird. Nachdrücklichst wird gegen die zu häufige Ausführung der Retro-flexionsoperation protestirt, indem darauf hingewiesen wird, dass bei beweg-lichen Retroflexionen die Beschwerden, wenn überhaupt vorlianden, vielfach von den begleitenden Umständen, bei fixirter R. von den entzündlichen Veränderungen der Nachbarschaft abhängen. Es sclieint fast, als ob schliess-lich nur die Ventrofixation als gelegentlicher Eingriff nacli ausgefûhrter Adnexoperation anerkannt wird. Bei der Anatomie der Gynatresieen wird die neuerdings von Nag el und J. Ye it vertretene Anschauung, dass ein grosser Teil der angeb-lich angeborenen Atresieen erworben sei, angeführt, die Gründe Veit's, namentlich bezüglicli der einseitigen Gynatresieen bei Verdoppelung des Genitalschlauches aber als nicht stichhaltig bezeielmet, da besonders adhaesive Peritonitis aucli ohue Infektion sich ausbilden könne, ferner $\backslash \tau \imath$-fektion auch bei geschlossenen Blutsäcken nachträglich vom Darin her ein-treten könne. Far die Entstehung der Blutsäcke in den Tuben wird die Sänger'sche Erklärung herangezogen (Zurückfliessen des Blutes aus dem überfüllten Uterus dureh die Tube in die Bauchhöhle, als Folge adhaesive Peritonitis und Verschluss der Tuben).

Urinfisteln. Bezüglich der Freilegung der Eisteln werden die Methoden von Fritsch und Schauta zur Loslösung von mit dem Knochen verwachsenen Fistelrändern als zweckmässig erwälmt, die Methode von Frank (Voroperation zur Erzeugung eines künstlichen Prolapses) als in ihrem Nutzen problematisch bezeichnet. Die grossen Vorteile, $\Lambda$ velche die Methode der Ñarbenspaltamg und Lappenbildung für die Anfrischung $1 \cdot$ rachten, werden hervorgehoben, auf die verschiedenen Modificationen weniger $\Lambda$ Vert gelegt. Von den komplízirteren hierher gehörigen Verfahren findet besonders das von Bardenheuer (extraperitoneales Vorgehen von einem suprasymphysären Sehnitt aus) und Tren delenbur g (Anfrischung von der Blase aus nach Sectio alta) $\Lambda$ Vürdigung. In den Fallen von weit-gehender oder totaler Zerstörung der Harnröhre ist die Herstellung der Kontinenz durch plastische Operationen noch imnier nicht mit einiger Sicherheit zu erreichen: es kommt die vollständige Verschliessung der unteren Ausflussöffnung und die Anlegung einer suprasymphysären Ausfluss-öffnung nach $\Lambda$ Vitzel in Frage.

Bei der Operation der Blasengebärmutterfistel wird die Methode der Loslösung der Blase vom Uterus und der isolirten Vernähung des Blasendefekts in den Yordergrund gestellt, bei Harnleiterfisteln Mackenrodt's Verfahren der directen Implantation eines die Ureterfistel umfassenden Scheidenlappens in eine zu diesem Zwecke angelegte Blasen-wunde (bei Gebärmutterharnleitertistel nach vorheriger Umwandlung der-selben in eine Scheidenfistel). Prolaps. Die Kolpoperineorhaphie in ihrer Bedeutung als Haupt-operation $\Lambda$ vird aufrecht erhalten und gegenüber Küstner und Macken-rodt sehr entschieden festgestellt, dass man Dauerheilungen von Prolapsen auch ohne gleichzeitige Inangriffnahme der Retroversio-tlexio 
fast immer merzielen kann. Am meisten von den in neuerer Zeit angewandten Methoden wird auch hier der Ventrofixation zugetraut. Bei der als Hülfsoperation häufig ausgeführten Elythrorhaphia anterior wird der Scheidenlappen meist als Falte in einem von He gar modifizirten Klemmapparat gefasst und nach Anlegung der Nähte unter demselben abgetragen. Auf die Fehling'sche doppelte Anfrischung wird kein besonderer $\Lambda$ Vert gelegt, Von den älteren Prolapsoperationen wird die M.artin'sche Anfrischung (zu beiden Seiten der Col. rug.) noch entschiedener als früher für ungeeignet bei Prolaps erklärt, das Bisch off'sche $\Lambda \tau$ erfahren als obsolet; es wurde seit Jahren nicht mehr angewendet. Bei der Besprechung neuerer Methoden wird die Kolpoperineorhaphie nach A. R.Simpson (Bildung zweier dreieckiger Scheidenlappen mit oberer Basis, welche nach oben geschlagen und nach der Scheide zu quer vereinigt werden; da $\pi$ ter quere Vereinigung der Dammwundo) als vorzüglich verwertbar zur Heilung grosser Scheiden- und Gebärmutter-vorfälle hervorgehoben, während die Frank'sche Methode der Dammspaltung mit nachfolgender querer Vereinigung verworfen wird, ein Urteil, das Buchanzeigen.

455

Mánchem vielleiclit nicht ganz verständlich erscheinen wird, da das Prinzip beider Operationen doch im $\Lambda$ vesentlichen das gleiche ist. Zudem verwendet Hegar selbst bei der Simp son'schen Anfrischung die bei der Frank'schen getadelten versenkten Catgutnähte, bei ersterer fäílt ebenso wenig Gewebe weg, wie bei letzterer, nur bei dieser aber wird das als kein thatsächlicher Vorteil ausdrücklich bezeicb.net. Bezüglich der Complikationen nach Prolapsoperationen ist ein Fall yon Interesse, in welchem Ileusersclieinungen auftraten, die nach Entfernung der Nähte am dritten Tage verschwanden; er wird aus dem Mitfassen eines Appendix epiploicus oder eines Netzstückes @durch die während des Auftretens von Brechbewegungen der Patientin an-gelegten obersten Nähte erklärt. Die Resultate sowohl in Bezug auf prima intentio, als auf dauernde Heilung waren sehr günstig. Recidive -•wurden nur ganz ausnahmsweise bekannt.

In einem Anhang wird noch die „Cysto pexie’ · bei schweren Pro-lapsen mi.t hochgradiger Cystocele besonderer Beachtung empfohlen; sie wurde sechs Mai von der eröffneten Bauchhöhle aus ausgeführt, ausgedehnte Plastiken angeschlossen; fünf Mai wurden so hervorragend günstige Heil-erfolge erzielt.

Der Exstírpation der Vagina wird ein besonderes Kapitel ge-widmet und dabei der Methoden von Olshausen, Dührssen, Thomas und anderer gedacht.

Die Operationen des veralteten Dammriss es werden eingetheilt in 1) Anfrischungs-, 2) Lappen-, 3) reine Spaltungsmethoden (ausserdem noch verschiedene Kombinationen dieser Verfahren).

Das grundsätzliche Weg-schneiden alien Narbengewebes ist durchaus nicht nötig und demzufolge wird den darauf besondere Rücksicht nehmenden Anfrischungen von Freund und Küstner wenig Wert beigelegt; auch die Tait-Saeng er'sche Methode der Dammspaltung erfährt geringes Lob: der Vorzug, dass der Verlust an Gewebe vermieden $\Lambda$ vird, sei iliusorisch und die Angabe, dass das Verfahren die natürlichen anatomischen Verhältnisse am vollkommensten wiederherstelle, nicht gerechtfertigt. Nur bei ganz einfachen Verhältnissen und geringem Dammdefekt hat die Methode wegen der sehnellen und leichten Ausführbarkeit Vorzüge, ihr Hauptgebiet ist also (entgegen Saenger) der inkomplete, nicht der komplete Dammriss. Noch einmal wird Gelegenheit genommen, Bedeuken zu äussern gegen allzu ausgedehnte Anwendung fortlaufender Catgutnähte, obgleich dieselben auch in Freiburg gelegentlich benützt werden; insbesondere das Heraus-leiten eines zusammenhängenden Fadens von der Schleimhautoberfläche in die Tiefe der Wunde als chirurgisch unrichtig bezeichnet. Als Hauptanfrischungsmethode $\Lambda$ vird die alte $\mathrm{S}$ imón-Hegar'sehe beschrieben, die nach wie vor 
durchgehends günstige Erfolge ergab. Die Durchschneidung des Sphincter ani zur Entspannung wurde als entbehrlich seit 10 Jahren nicht mehr geübt. $\Lambda$ on den Lappenmethoden, deren Prinzip der Vermeidung von Gewebsverlust gerade beim kompleten Dammriss grosse Bedeutung haben kann, hat besonders die Methode von A. R. Simpson (Bildung von je zwei Scheiden und Mastdarmlappen von einem auf der Narbe verlaufenden Bogen-schnitt aus) mit einer kleinen Modification des Querschnitts und der Ver-wendung versenkter Catgutnähte, vorzügliche Dienste geleistet und wurde in den letzten Jahren fast ausschliesslich benützt. Auch die eine reine Spaltungsmethode darstellende, totale Dammplastik nach Fritsch wird als rationell hervorgehoben.

Der vorliegende Bericht konnte nur in dem eingangs erwähnten Sinneauf die wichtigsten Punkte aufmerksam machen: es ist klar, dass derselbekeinen Begriff geben kann von dem Genuss und dem Gewinne, welche eiriVertiefen in das Buch selbst auch in seiner neuen Form jedem Arzte ge- $\Lambda$ vähren wird. $\quad 0$. von Franqu é-Würzburg.

Monats $\beta$ chríft für G-eburtshülfe und Gynaekologi $\theta$. Bd. VI. Heft i. 31

456

Buchanzeigen.

F. von Winekel, Behandlung der von den w eiblich en Genitalien ausgehenden Entzündungen des Bauchfelles und desbenach-barten Zellgewebes. I. Suppl.-Bd. 2. Heít des Handbuchs der spec. Therapie inn. Krankheiten. Peuzoldt-Stintzing. Jena 1897. Mit 9 teil-weise farbigen Abb. im Text. S. 77-145.

Es ist überaus dankenswerth, dass der hochverdiente Müncliener Kliniker in dieser brennenden Frage das Wort ergriffen hat. In der ihm eigenen klassischenWeise hat er das einschlägigeMaterial zusammengetragen und kritisch gesichtet, $\mathrm{nm}$ das, was sich bis heute an therapeutischen Sätzen als gesichert herausgestellt hat, an der Hand seines eigenen klinisehen Materials praegnant darzustellen. F. v. $\Lambda$ Vinckel hat sich selbst auf dem Moskauer internat. Med. Congress mit grosser Reserve über das heute als sicher ge-wonnene therapeutische Ergebniss ausgesprochen: aber unzweifelhaft hat er mit sicherer Hand das bisher weit zerstreute Grundmaterial so übersichtlich

$\mathrm{f}$

eordnet und so bedeutungsvolle Beiträge aus seiner reiçhen Erfahrung erbeigebracht, dass hier die weitere Bearbeitung dieses so wichtigen Capitels ansetzen kann.

Die Darstellung der $\Lambda \tau$ erhältnisse des Beckenbindegewebes stützt sich auf Winckel's eigene Präparate; dieselben haben durch die inzwischen bes. von A. v. Rosthorn auf dem Leipziger Gynäkologen-Congress demonstrirten eine, soviel wir sehen konnten, sehr bestimmte Bestätigung gefunden. $\Lambda$ Vinckel sondert die Entzündungen des Beckenzellgewebes von denen des Becken-bauchfelles und anderen allgenieinen Bauchfellentzündungen. Unzweifelhaft muss diese Eintheilung der Betrachtung dieser von den Genitalien ausgehenden Entzündungsprocesse zu Grunde gelegt werden, $\Lambda$ venn auch in der Praxis - wie $\Lambda \mathrm{V}$. das selbst hervorhebt - dieselben nur zu häufig sich ver-gesellschaften.

Mit vollem Recht hebt er hervor, dass die Parametritiden heute stets als infectiösen Ursprungs angesehen $\Lambda$ verden müssen, und dass nur die Arten und Combinationen der Mikroben. ihre Menge und Virulenz variiren. Es ist überraschend, dass diese Processe nur in 2 pCt. der Fälle in Vereiterung übergehen: sicher entziehen sich die minder schweren nicht selten der ärzt-lichen Kontrolle. Mir will scheinen, dass die Fälle der Freund'schen atrophicirenden Parametritis nicht ganz so selten sind, wie $\Lambda$ Vinckel es darzustellen scheint, während wir in der That zugeben müssen, dass die Adenitis periuterina uns auch in unserm immerhin erheblich gewachsenen 
Untersuchungsmaterialnursehrvereinzeltvorgekommen ist. Die Schwierigkeiten der Differentialdiagnose $\Lambda$ vird jeder Fachmann wohl zu schätzen wissen; für den Praktiker, dem doch dieses Handbuch die $\Lambda \Lambda \tau$ ege weisen soil, wären vielleicht weitere Details erwünscht gewesen.

Unter den therapeutischen $\Lambda \tau$ orschlägen ist sicherlich alles erörtert, was hier in Frage kommt. $\Lambda$ Venn Verf. an dieser und auch einmal an einer späteren Stelle sich gegen die Blutentziehung durch Blutegel am Unterleib ausspricht, dabei Referenten als einen Befürworter dieser Behandlungsweise hervorhebt, so möchte sich letzterer doch zu bemerken erlauben, dass, wenn jene Empfelilung nicht der jüngsten Vergangenheit entspricht, sie doch nach seiner Meinung auch heute noch nicht ganz von der Hand zu weisen ist und gelegentlich mindestens schmerzlindernd wirkt, wo die Eisbehandlung diesen Ziel nicht voll erreicht. Das von Frits ch empfohlene Verfahren der Eröffnung parametritischer Abscesse, dem auch Verf. das $\Lambda$ Vort redet, ist uns weit umständlicher und in nichts sicherer erschienen, als die von uns stets geübte unmittelbare Entleerung durch einen Trokar, dessen Einführungskanal stumpf oder schneidend derart erweitert wird, dass Ausräumung und Drainage der Höhle ausgeführt werden kann: ev. dabei verletzte Gefässe konnte Ref. stets leicht umstechen.

Die Verwerfung der Anwendung des galvanischen Stromes zur Be-förderung der Rückbildung ist wohl fast allseitiger Zustimmung sicher. -

$\Lambda \tau$ erf. berechnet die Häufigkeit der Perimetritis zu nahezu 80 pCt.; nach des Ref. Erfahrung dürfte diese Zahl durchaus den Thatsachen ent-sprechen, besonders wenn die Pigmentflecken im Perimetrium und die Pachy-Peritonitis haemorrhagica mit einberechnet $\Lambda$ verden. Ausser den von $\Lambda V$. angeführten Befunden bei Perimetritis hat Ref. relativ häufig herpes-

Buchanzeigen.

457

ähnliche Bläschen in dem. Perimetrium gesehen und -wirklich pulpöse zottige allgemeine Verdickung, $\Lambda$ vobei es aber auffallender Weise nur zu partieller Verwachsung mit Nachbarorganen gekommen war.

TJnter den Symptomen wird hier ebenso wie bei der Parametritis auf die Tussis uterina hingewiesen: wir konnten dieses auch uns auffallende Symptom gelegentlich aber doch nur der Neurasthenie und Hysterie zu-sc·hreiben, welche allerdings eine nicht seltene Folge der chronischen Beckenbauchfell- und Beckenzellge $\Lambda$ vebsentzündung ist. Mehr Gewicht mussten wir auf die Sehstörung legen, auf welche Mooren näher eingegangen ist. Wir haben wiederholentlich Gelegenheit gehabt, mit Mooren derartige Fälle gemeinsam zu sehen und uns von der Bedeutung dieses Folgezustandes chronischer Beckenentzündungen zu überzeugen. Es stimmt vollständig mit unseren eigenen Erfahrungen überein, dass die Prognose der chronischen Perimetritís weniger günstig ist als die der Parametritis.

$\mathrm{Zu}$ der Punction perimetritischer Ergüsse haVjen wir nur sehr selten Veranlassung gefunden. Die Bedenken gegen das Schultz e'sche Verfahren zur Sprengung perimetritischer Verwachsungen hätten wir gern etwas starker hervorgehoben gesehen; wenn in der Hand Erfahrener, über alle Mittel klinischer Fürsorge Gebietender gewiss die gefahrvollen Folgezustände nach derartigen Manip $\pi$ lationen unter Controlle gehalten werden können, so können $\lambda$ vi $\gamma$ doch nicht umhin zu besorgen, dass in der Hand Ungeübter recht sehlimme Erfahrungen dabei gemacht werden; einige Beispiele aus der consultativen Praxis berechtigen uns zu dieser Bemerkung.

Die Castratio uterina wird mit vollem Recht als Ultimum Refugium hingestellt: unsere eigenen Erfahrungen bestätigen das vollauf. 
Die allgemeine Beckenbauchfellentzündung wird je nach ihrer puerperalen, post, operativen, gonorrhoischen, tuberkulösen und nach der Entstehung aus der Perforation von Unterleibsorganen gewürdigt. Die Arbeiten von Kroenig, Menge und $\Lambda$ Vertheim finden ihre voile Be-rücksichtigung. W. führt an, dass Menge in 10 pCt. der Eitertaben den Bacillus tuberculosis gefunden habe. Nach unseren Erfahrungen darf daraus kein Schluss auf die Häufigkeit der Tuberculose des Peritoneum ge-zogen werden.

Sehr beachtenswerth erscheinen die Versuche, aus dem kliníschen Bild die Anhaltspunkte für die Beurtheilung der Aetiologie der einzelnen Er-krankungsformen zu gewinnen; einen Theil unserer eigenen Fälle konnten wir gut in die einzelnen Rubriken eiiiregistriren, doch war für eine Anzahl anderer der Symptomencomplex zu wirr oder zu unbestimmt, sodass gewiss erst weitere Erfahrungen hier Klarheit bringen werden.

Winckel selbst hebt hervor, dass die Indikationen der operativen Behandlung durch das vorliegende Material noch nicht definitiv entschiedera werden. Für die puerperale, progrediente diffuse Peritonitis sollte, nach den glänzenden Erfahrungen der Operation bei eitrigen PleuraExsudaten, die Hoffnung auf gute Erfolge bei ähnlichem Yorgehen berechtigt erscheinen. Nach den bisher veröffentlichten Fallen ist das Resultat ( $21 \mathrm{pCt}$. Genesungen) schlechter als das der spontan genesenen Fälle (26,4 pCt.). Sicher ware es verfehlt, daraus Schlüsse zu ziehen. Weitere Erfahrungen müssen erst die Entscheidung bringen. Den mitinncirten Uterus ohne Weiteres mitzuent-fernen halten wir mit Winckel für unmotivirt, da in solchen Fallen mit ihm keineswegs alle Schädlichkeiten und Eiterheerde entfernt werden. Unheil-bar erkrankte Ad $\pi$ exmassen müssen entfernt werden; gegen die Entternung des Uterus kommt nach unserer Erfahrung noch in Betracht, dass damit unzweifelhaft die Bedeutung des operativen Eingriffs gewaltig gesteigert wird.

Gewiss liegt theoretisch kein Grund vor, die Behandlung der Peritonitis post operationem, die Entfernung des Eiters durch eine Wiedereröffnung der Bauchhöhle nicht als rationell zu bezeichnen; leider lehrt die Erfahrung, dass der Erfolg nur zu häufig ausbleibt. Das Warum? ist zur Zeit noch unerfindlich. Ob die Empfehlung des Verf.: nach Entleerung des. Eiters zu drainiren, günstigere Resultate zeitigen wird, bleibt abzuwarten. Ref. hat den Muth hierzu noch nicht wieder gefunden.

Die gonorrhoische Peritonitis erfordert nur selten die Erüffnung der

$31^{*}$

458

Buchanzeigen.

Bau'chhühle; dann würde besonders der Ausgangspunkt zu entfernen sein, auf vaginalem oder abdominalem Weg. Dieser Satz Winckel's dürfte ebenso wie der beziiglich der Beliandlung der tuberkulösen Peritonitis wohl all-seitiger Zustimmung sicher sein; Koeliotomia abdominalis. Mit volleni Recht betonte W., dass man Heilesultate erst in í'ünf Jahren feststellen solle; erst dann ist Genesung als sicher anzusehen.

Den Eingrifi bei Perforationsperitonitis will $\Lambda \mathrm{V}$. - und darin stimmen wir ihm gewiss alle bei je nach den Ursachen verschieden gestalten. Während die serös-fibrinös eitrigen, nicht jauchigen Exsudate. Sactosalpingen, Ovarialtumoren, incl.derDermoide, noch Aussichten bieten, erscheinen nachdem heutigen Standpunkt unserer Kunst die Pälle an Perforation jauchiger Abscesse unrettbar, selbst $\Lambda$ venn rasch und sicher aseptisch operirt wird.

In seinem Schlusswort empfiehlt Win eke I die Koeliotomia abdominalis zur Entleeruug massiger Exsudate, die Koel. vag. post, wenn sich die Exsudate im Douglas finden. Liegen hier 
massige Yerwachsungen, während die Plica vesico-uterina í'rei ist, dann giebt W. Kolpot. ant. den Vorzug.

Mit besonderer Genugthuung schliessen wir uns der Empfehlung $\Lambda$ Vinckel's an, die Operation so conservativ wie möglich zu gestalten. Winckel spricht sich als erster deutscher Kliniker rückhaltlos dafür aus, ev. auch kleine Theile der Ovarien zu erhalten und atretische Tuben zu öffnen, wofür wir so lange, ohne die voile Zustimmung unserer Fachgenossen zu finden, gekämpft haben. Auch für Winckel ist die Radikaloperation (Entfernung auch des Uterus) den sehr seltnen Fallen ausgedehntester Jauchung des Uterus und anderweiter schwerer Erkrankung desselben, den Fallen von recidivirender, chronischer, diffuser und lokaler Peritonitis mit Durcheiterung des kleinen Beckens vorzubehalten.

Das vorliegende Buch ist ein Merkstein auf dem Entwicklungs $\Lambda$ vegeder Behandlung der so schwer zugänglichen Entzündungsprocesse im Becken.Yon ihm wird weitere. fruchtbringende Anregung ausgehen: es ist ein rechtesWort zur rechten Zeit! A. Martin. Franz Ludwig Neugebauer, Die Fremdkörper des Uterus. Zusammenstellung von 550 Beobachtungen aus der Litteratur und Praxis.

Breslau 1897. Preuss u. Jünger.

Arbeiten, $\lambda v i \theta$ die vorliegende, nennt man corapilatorische. Dieser Richtung wissenschaftlicher Thätigkeit etwas Geringschätziges anzuhängen, ware etwa ebenso verkehrt und ungerecht, als wollte man beispielsweise der historischen Quellenforschung, der Litteraturgeschichte und noch mancher anderen Disciplin deswegen den Titel Wissenschaft versagen, weil nicht Eigenes gebracht, sondern Fremdes zusammengestellt und commentirt wird. Auch wir kõnnen die compilatorische Sammelarbeit nicht entbehren und, wenn wir etwas bedauern, so ist es, dass wir nicht noch mehr Leute haben, welche auf diesem schwierigen und entsagungsvollen Gebiete Kraft und Zeit opfern, wie Neugebauer der Jüngere, welcher vermöge einer gewissen hereditären Begabung und Neigung dermalen geradezu als Alleinbeherrscher auf demselben dasteht. Man stelle sich nur vor, dass alle casuistischen Mittheilungen in gleicher Art gesichtet und zusammengestellt würden, wie es Neugebauer nun schon für mehrere Gruppen soldier in mustergültiger Weise gethan hat, um zu ermessen, welcher Vortheil für ihre Ausnutzung und weitere wissenschaftliche Verwerthung gewonnen würde.

Wer nun meint, in dem neuesten Werke Neugebauer's eine end-lose und eintönige Aufzählung einiger Hunderte von Fallen zu finden, wird überrascht sein, zu bemerken, in $\Lambda$ vie geschickter $\Lambda$ Veise das riesige Material sortirt und nach klinischen Gruppen derart übersichtlich geordnet ist, dass jede derselben leiclit auffindbar ist und neue Beobachtungen bequem eingereiht werden können. Dies wird auch noch weiter ermöglicht durch ein Inhalts-verzeichniss mit abgekürzter Angabe der Besonderheit des Einzelfalles.

Neugebauer theilt das Gebiet der Fremdkörper des Uterus in folgende acht Gruppen :

I. Von aussen her in die Uterushöhle absichtlich eingeführte oder zu-fällig eingedrungene Fremdkörper, Pessarien, Instrumente, Sonden, allerhand behufs Aborteinleitung und Masturbation eingeführte Fremdkörper.

Buchanzeigen.

459

II. Parasiten in der Uterushöhle: EcL·inococcus u. s. Iv.

III. Aus dem Körper auf dem $\Lambda$ Vege der Perforation der Uteruswandin die Uterinhöhle eingetretene Fremdkörper: Faeces, Harn, Eiter, Productedurchgebrochener Neoplasmen, Ovarialeysten, Extrauterinschwangerschaften, parametrische Abscesse. 
IV. Nach Uterusruptur oder instrumenteller Perforation in den Uteruseingetretene Darmschlingen.

$\Lambda \Gamma$. In der Uterushöhle retinirte Secrete nnd deren Producte bei Haematometra, Lochiometra, Hydrometra, Pyometra und die Gasansammlung bei Physometra.

VI. Vom Uterinmutterboden gebildete und theilweise gelösteNeoplasmenund deren Verkalkungsproducte, die sog. Uterussteine.

VII. Die sog. Haargeschwülste des Uterus.

VIII. Retinirte Schwangerschaftsproducte bei missed abortion, missedlabour, Zurückhaltung abgestorbener Früchte, einzelner Fruchttheile, derPlacenta, Eihäute u. s. w.

Man sieht, Neugebauer fasst den Begriff „Fremdkörper des Uterus” in einem sehr weiten Sinne; doch wird man darüber gewiss weniger rechten, als $\Lambda$ venn es umgekehrt ware.

Die V. Gruppe wird allerdings nur in der Eintheilung aufgeführt. Man kann Neugebauer gewiss nur zustimmen. dass sie besser von einem andern Standpunkte . aus betrachtet werde. Auch hat er die Casuistik des Eintretens von Darmschlingen in den Uterus nach Ruptur sub partu noch nicht zusammengestellt.

Die grösste Gruppe ist die VIII., Retention von Schwangerschafts-producten. Sie umfasst, die Fälle von 180-550, abgerech.net eine Anzahl Nummern von Nachträgen für die anderen Gruppen. Das Kapitel .,Missed abortion” zählt allein 260 Beobachtungen. - Sehr dankens $\Lambda$ verth ist auch das Schlusskapitel über Spätgeburt - Partus serotinus - mit 36 neueren Beobachtungen, die unter die 550 Gesammtfälle nicht inbegriffen sind.

Diese wenigen Hinweise werden genügen, urn mit Freuden zu erkennen, welchen werthvollen Besitz $\Lambda$ vir in diesem neuesten mühevollen, erstaunlich fleissigen $\Lambda$ Verke Neugebauer's gewonneu haben. In keiner Fachbibliothek sollte es fehlen, und auch um deswillen sollte seine Anschaffung eine recht all-seitige werden, weil solche $\Lambda$ Verke von den Verlegern nicht gern über-nommen werden und auch ein buchhändlerischer Erfolg neben dem unbe-stritten litterarischen eine Aufmunterung für den Autor ware, dem, im besten Sinne, compilatorischen Arbeiten treu zu bleiben.

S ä n g e r. A. CzempiП. Die Technik der Ohio roformnar cose für Aerzte und Studirende. Berlin 1897. Otto Enslin.

Das Hauptgewicht bei der Technik der Chloroformnarcose liegt in der sorgfältigsten Beobachttmg des Pupillarreflexes. Beim Beginn der Narcose erfolgt zunächst eine Erweiterung der Pupille, die auf Lichteinfall deutlich reagirt. Allmählich tritt mit dem SchwindeD des Bewusstseins eine Verengerung der Pupille ein, bis bei intensiver werdender Narcose der Normalpunkt erreicht wird. Dieser ist charakterisirt durch die gänzlich verengte reactionslose Pupille. Schmerzempfindung und Muskel-thätigkeit sowie Bewusstsein sind vollständig aufgehoben, andererseits be-steht keine Gefahr für das Leben des Narcotisirten. Lässt man jedoch über diesen Punkt hinaus weiter Chloroform einathmen, so erfolgt eine Erweiterung der Pupille, welche alsdann auf Licht nicht mehr reagirt. Die Pupille erweitert sich ad maximum, die Athmung wird oberflächlicher und hört schliesslich ganz auf, das Herz steht still und der Tod tritt ein. Diese einzelnen Phasen der Narcose kennzeichnet der Verf. sehr übersicht-lich auf einer beigegebenen Tafel.

Auf einer horizontalen Linie steht links am Anfang das Erwachen, rechts am Ende der Tod. Etwa in der Mitte, doch etwas mehr nach rechts gelegen ist der Normalpunkt. In einer gewissen Entfernung davon nach links befindet sich die weite Pupille mit Reflex, nach rechts die weite reactionslose Pupille. Ein gewisser Raum, der gleichmässig vor und hinter dem Normalpunkt gelegen ist. wird als Narcosenbreite bezeichnet. 
460

Buchanzeigen.

Diese ist sehr wechselnd und verschieden bei den einzelnen Individuen. Es ist die Aufgabe des Narcotisirenden, sich rasch und sicher über die Narcosenbreite des Patienten zu orientiren, in welcher er denselben wälirend der ganzen Nareose zu erhalten bemiiht sein muss. Ein aufmerksamer Beob-achter kann jederzeit an dem Pupillarreflex erkennen, in welchem Stadium der Nareose sich der Chloroformirte befindet.

$\Lambda$ Veniger Werth ist auf den Cornealreflex zu legen, welcher nor-maler Weise zwar am Normalpunkt erlosclien ist, ausnahnisweise aber auch darüber hinaus fortbesteht und zu Täuschungen Anlass geben kann. Ausser-dem aber ist auch das öftere Prüfen desselben für die Cornea schädlich und der Cornealretlex selbst nicht immer deutlich.

Nächst der Pupillenreaction ist während der ganzen Nareose die Athmung des Narcotisirten genau zu beobachten. Gestört wird diese hauptsächlich durch schlechte, unpassende Lagerung des Kopfes, Be-hinderung der Nasenathmung, Zurückfallen der Zunge, Anwesenheit von Flüssigkeit im Nasen-Rachenraum etc.

Die Ursachen der behinderten Athmung sowie deren Vermeidung und Beseitigung werden näher beschrieben. 1st durch Ueberschreiten der Narcosenbreite Athmungsstillstand eingetreten, so kann der Patient noch durch Zuführen von frischer Luft, durch künstliche Athmung gerettet werden. Bei Herzstillstand ist eventuell Herzmassage zu versuchen.

Den Puls des Narcotisirten zu prüfen, ist bei normal verlaufender Nareose kaum nöthig. Es empfiehlt sich, vor der Nareose sich über die Qualität des Pulses zu orientiren, um bei Zwischenfällen während der Operation dessen frühere Beschaffenheit zu kennen.

Ist der Patient rechts vom Normalpunkt angelangt, also übernarcotisirt worden, so ist am Pulse zu prüfen, ob bereits ein Herzstillstand oder nur ein Athmungsstillstand stattgefunden hat. $\mathrm{Zu}$ achten ist auch auf den Puls bei Beginn der Nareose wegen Gefahr einer Herzlähmung in diesem Stadium, sowie ferner bei grossen Blutverlusten während der Operation und bei Ein-griffen, welche starke Shockwirkungen hervorzurufen geeignet sind.

Der Tod in der Nareose kann eintreten durch Vergiftung mit Chloroform oder durch Insufficienz der vitalen Organe des Patienten. Die Vergiftung kann stattfinden durch eine Ueberdosirung mit Chloroform oder indirect veranlasst durch behinderte Athmung in der Nareose, in beiden Fallen also durch Unachtsamkeit oder durch ITnkenntniss des Narcotisirenden. Verf. glaubt, dass ein grosser Theil der Chloroformtodesfälle in diese Kate-gorie gehört.

Eine andere Todesart, der sogenannte Herztod, in der Nareose kann ebenfalls oft, wenn auch nicht immer durch gute Technik bei Beginn der Nareose vermieden werden.

Nicht der Chlorofornmarcose, sondern der Insufficienz der vitalen Organe sind die Todesfälle in der Nareose zuzuschreiben, wenn Verletzungen oder anhaltende Krankheit den Gesammtorganismus so geschwächt haben, dass der Tod auch ohne Nareose stündlich zu erwarten war. Das Narcoti-cum kann allerdings dabei manchmal beschleunigend wirken. Ebenso ist auch der Tod nach der Nareose aufzufassen. 